Methods Sixty-five patients with ACS and 75 controls with SAP with similar atherosclerotic risk profiles were studied. CT angiography was performed using a dual-source CT scanner before invasive catheterisation. The lesion characteristics that were assessed included luminal cross-sectional area (L-CSA), vascular cross-sectional area (V-CSA), plaque area, and degree of stenosis with DSCT and quantitative coronary angiography (OCA), and plaque types, mean and minimal CT density (HU), remodelling index, and the presence of 'spotty' calcifications with DSCT.

Results All parameters, including L-CSA, V-CSA, plaque area, and degree of stenosis showed a good correlation between DSCT and QCA $(p<0.05)$. In comparison with stable lesions in SAP, culprit lesions in ACS had a much larger mean V-CSA (20.5 \pm 6.0 vs 14.8 $\left.\pm 4.8 \mathrm{~mm}^{2}\right)$, plaque area $\left(15.3 \pm 5.0\right.$ vs $\left.11.1 \pm 3.3 \mathrm{~mm}^{2}\right)$, and remodelling index $(1.3 \pm 0.2$ vs $1.0 \pm 0.4)(p<0.05)$. The prevalence of noncalcified/calcified/mixed plaque was $30 / 0 / 35$ for culprit lesions in ACS compared with $25 / 15 / 35$ for stable lesions in SAP $(p<0.01)$. The proportion of 'spotty' calcified plaque was $21.5 \%$ in ACS culprit lesions (14 of 65 ) compared with $1.3 \%$ for stable lesions in SAP (1/75). The mean HU and minimum HU of culprit lesions in ACS versus those in stable lesions of SAP were $88.6 \pm 43.2$ vs 154.2 $\pm 98.7(p<0.01)$ and $45.9 \pm 34.7$ vs $98.2 \pm 76.8(p<0.01)$, respectively.

Conclusions DSCT is feasible to quantify plaque and distinguish culprit lesions in ACS, which display a greater proportion of noncalcified material and 'spotty' calcifications, lower CT attenuation, and a higher remodelling index compared with stable lesions in SAP.

\section{GW23-e0452 CHARACTERISATION BY DUAL-SOURCE CT OF CULPRIT LESIONS IN ACUTE CORONARY SYNDROMES COMPARED WITH STABLE ANGINA PECTORIS}

doi:10.1136/heartjnl-2012-302920ad.28

yang xia, chen yundai. The Chinese Pla General Hospital

Objectives The advent of dual-source CT (DSCT) has enabled easy detection of atherosclerotic plaques and assessment of their composition and mechanical properties. This study assessed the value of DSCT in identifying the characteristics of culprit lesions in acute coronary syndrome (ACS) as compared with lesions instable angina pectoris (SAP). 